\title{
Formation of 2D structures and their transformation by external stimuli: a scanning tunneling microscopy study
}

\author{
Yoshihiro Kikkawa \\ We present a review of our recent research on the formation of two-dimensional (2D) nanopatterns via self-assembly and their \\ transformations by external stimuli. The proper design and synthesis of functional molecules and the ability to follow their \\ integrations on a surface would allow the effective use of their nanofunctions to produce novel nanomaterials and devices. \\ In this context, we synthesized stimuli-responsive molecules that contain alkyl chain units, which act as not only the \\ adsorption unit onto the substrate but also as the intermolecular interaction site. The 2D molecular arrangements were then \\ directly visualized using scanning tunneling microscopy at the solid/liquid interface. Thermal treatment, metal coordination \\ and ultraviolet irradiation induced chemical structural conversions of the individual molecules, which resulted in the \\ transformations of the 2D structures.
}

Polymer Journal (2013) 45, 255-260; doi:10.1038/pj.2012.132; published online 27 June 2012

Keywords: metal coordination; photodimerization; scanning tunneling microscopy; self-assembly; solid -liquid interface; tandem Claisen rearrangement

\section{INTRODUCTION}

Controlled molecular self-assemblies and the subsequent alteration of their patterns on a surface enable the construction of molecule-based devices and circuits with post-tunable properties and functions. In nature, some systems show stimuli-responsive conformational and skeletal changes to achieve the functional transformation. For example, the acetylcholine receptor forms a tubular structure across a cell membrane, ${ }^{1,2}$ and the binding of acetylcholine onto the acetylcholine receptor induces a conformational change to open the ion channel, which allows the flow of positively charged ions across the membrane. The careful design and synthesis of functional molecules is a prerequisite for the development of well-organized functional molecular assemblies whose supramolecular structures are guided by non-covalent interactions, such as hydrogen bonds, van der Waals interactions, metal coordination, and so on. ${ }^{3-6}$

The molecular building blocks, sometimes called tectons, maintain their characteristic molecular shape, symmetry and interaction sites of non-covalent interaction, which results in the formation of various elaborate two-dimensional (2D) nanostructures. ${ }^{7-9}$ Among numerous non-covalent interactions, directional intermolecular hydrogen bonds and metal coordination have been introduced into tectons as the supramolecular scaffolds for the construction of various regular and stable 2D structures. ${ }^{10-13}$ Trimesic acid forms honeycomb networks, whereas terephthalic acid exhibits a linear columnar structure via hydrogen bonds. ${ }^{14}$ Another example is tectons with linear and bent backbones containing pyridine and terpyridine units as metal coordination sites that formed linear and zig-zag-shaped coordinating networks, respectively. ${ }^{15,16}$ The fabrications of other 2D structures, including grid and Kagomé structures, have been achieved through the synthesis of corresponding tectons with specific molecular shapes and interaction sites. ${ }^{17-19}$ In addition to the intermolecular interaction between adsorbates, adsorbate-solvent and adsorbate-substrate interactions have important roles to control the lateral and spatial arrangements of the building blocks on a surface. $^{20}$

Scanning tunneling microscopy (STM) is one of the most powerful scanning probe methods, and it enables the analysis of regular nanostructures at a molecular level. ${ }^{6,8-10,13}$ The tunneling current exhibits an exponential dependency on the distance between the probe tip and the samples on a flat conductive substrate, and the precise control of the distance by the feedback signal provides the high-resolution STM images. In the constant current mode, the probe tip scans and follows the sample surface at a constant tunneling current to obtain the topographic information, which reflects the physical height and electron density of the states. In the case of constant height mode, the vertical position of the probe tip is fixed, and the tunneling current is monitored as a function of lateral position.

Initially, STM was used for the observation of semiconductors, inorganic molecules and metal surfaces..$^{21-23}$ Isolated organic 
molecules have subsequently been targeted for analysis on a surface under vacuum at low temperatures. ${ }^{24}$ To overcome environmental limitations, 2D organic monolayers either chemisorbed or physisorbed on a conductive substrate were fabricated to adequately immobilize the molecules onto the substrate; these monolayers were subsequently observed under ambient conditions or at the solid/liquid interface. ${ }^{25-28}$ Particular to the solid/liquid interface system, highly oriented pyrolytic graphite (HOPG) has often been applied as the substrate because of its conductivity, flatness, cleanness, inertness and high affinity to alkyl chains, which act as the adsorption site of the compounds. As the lattice constants of HOPG are known to have $0.246-\mathrm{nm}$ periodicities, this value can be used as an internal standard for the correction of lateral dimensions by recording the submolecularly resolved STM images of the adsorbate and HOPG simultaneously. The physisorbed monolayers at the HOPG/solvent interface are easily prepared by simply dropping an aliquot of the sample solution dissolved in a non-conductive and preferably lowvolatility solvent, such as 1-phenyloctane, 1,2,4-trichloronenzene, octanol, and so on. ${ }^{8-10,20}$ The STM probe tip (mechanically cut Pt/ Ir) is then immersed in the deposited solution during the imaging. The spontaneously formed monolayer can be suitable for investigating

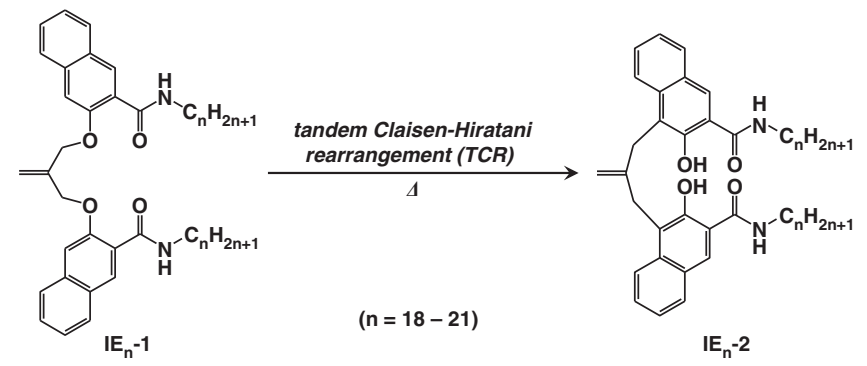

Scheme 1 Chemical structures of isobutenyl ether compounds before $\left(\mathrm{IE}_{n^{-}}-1\right)$ and after TCR (IE $\left.{ }_{n}-2\right)$. the relationships between the molecular structures and the resultant 2D molecular orientations. In addition, dynamic structural changes at the solid/liquid interface can be visualized in situ. ${ }^{11,29,30}$

Although a number of investigators have focused on the $2 \mathrm{D}$ structural formation using tectons, few studies can be found on the fabrication and control of nanoarchitectures by isotropic van der Waals interactions and post-structural conversions. This focused review provides a brief overview of our recent progress related to the formation of $2 \mathrm{D}$ structures by tuning the alkyl chain length and their subsequent transformation by external stimuli, such as heat, metallation and ultraviolet (UV) light. ${ }^{31-36}$ All of these 2D structures were visualized by STM at the HOPG/1-phenyloctane interface.

\section{THE ODD-EVEN EFFECT AND THE TANDEM CLAISEN REARRANGEMENT (TCR)}

Subtle changes in the intermolecular interactions between the adsorbates sometimes strongly influence the 2D structure formation. The odd-even effect is the well-known phenomena in which the alternate changes in the physical and chemical properties, as well as in the crystalline structures of a molecule, are observed to depend on the odd/even nature of the molecule's chain. ${ }^{37}$ Thermally responsive isobutenyl ether compounds that possess alkyl chains of different lengths were synthesized ( $\mathrm{IE}_{n}-1, n=18-21$; Scheme 1$)$ and the effect of the alkyl chain length on the 2D structure formation was investigated. ${ }^{31,32}$ Then, the $\mathrm{IE}_{n}-2$ compound was obtained through TCR (also called as Hiratani-double-Claisen rearrangement), ${ }^{38}$ which is a simple thermal reaction that can convert from ether to hydroxyl groups, accompanied by the formation of new C-C bond. ${ }^{39}$

Figures 1a-e show the STM images of isobutenyl compounds with various alkyl chain lengths. In the case of $\mathrm{IE}_{18^{-1}}$ (Figure 1a), periodical bright wavy lines and dark regions were observed. The wavy bright lines were composed of bright oval dots that correspond to the flat-on naphthalene units, which have a high tunneling efficiency. In the dark regions, the parallel straight lines of $2.1 \pm 0.1 \mathrm{~nm}$ and $1.4 \pm 0.1 \mathrm{~nm}$ were observed almost perpendicular to the bright line
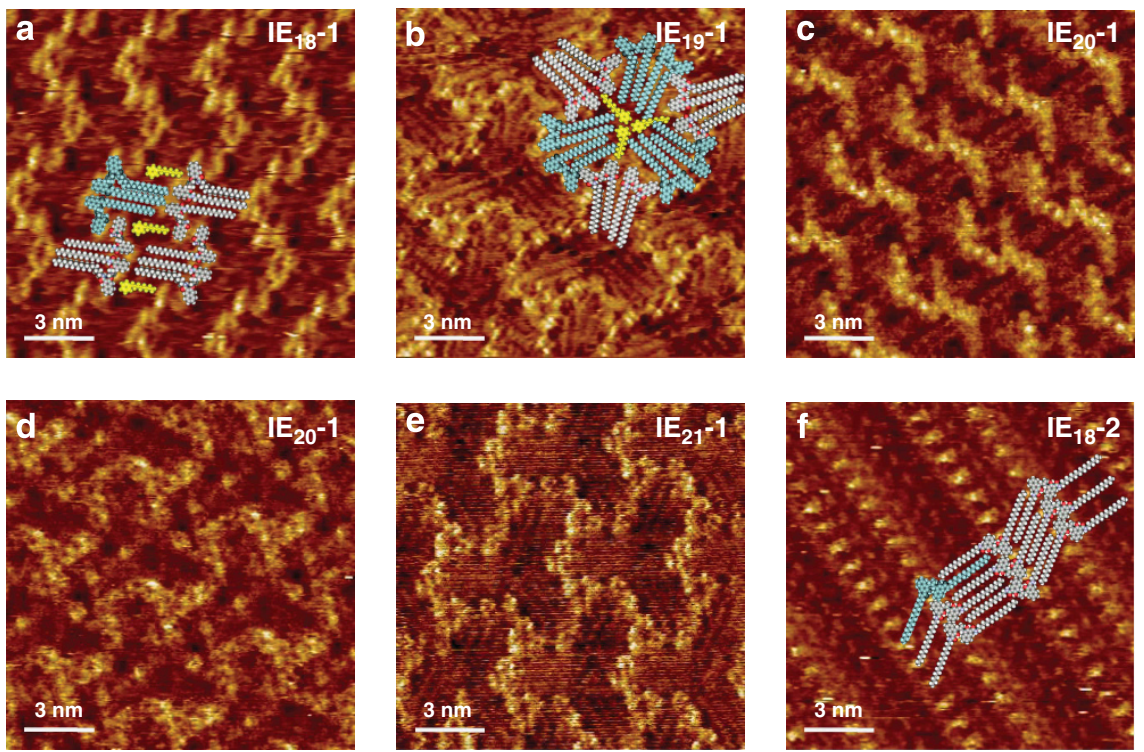

Figure 1 Typical STM images of $\mathrm{IE}_{n^{-1}}(n=18-21)$ and $\mathrm{IE}_{18}-2$ at the HOPG/1-phenyloctane interface. One of the building blocks and the coadsorbed 1-phenyloctane molecules are colored in light green and yellow, respectively. Molecular models are superimposed on each STM image. Labels and tunneling conditions: (a) $I \mathrm{E}_{18}-1, I=10 \mathrm{pA}, V=-616 \mathrm{mV}$; (b) $I \mathrm{E}_{19^{-}}-1, I=2.0 \mathrm{pA}, V=-604 \mathrm{mV}$; (c) $I \mathrm{E}_{20}-1$ (wavy structure), $I=2.9 \mathrm{pA}, V=-877 \mathrm{mV}$; (d) $I \mathrm{E}_{20}-1$ (tripod structure), $I=2.2 \mathrm{pA}, V=-985 \mathrm{mV}$; (e) $\mathrm{IE}_{21}-1, I=1.8 \mathrm{pA}, V=-957 \mathrm{mV}$; and (f) $I \mathrm{E}_{18}-2, I=3.9 \mathrm{pA}, V=-499 \mathrm{mV}$ after TCR. 
axis, which suggests that longer and shorter lines are derived from the octadecyl chain and the solvent molecule of 1-phenyloctane, respectively. Therefore, the basic skeleton of the wavy structure consists of a pair of molecules, where one of the four alkyl chains dangles into the solvent phase.

In the cases of $\mathrm{IE}_{19^{-}}-1$ and $\mathrm{IE}_{21}-1, \mathrm{C}_{3}$-symmetric tripod structures were formed. The framework is composed of the three pairs of the molecules, and all the alkyl chains are attached to HOPG (Figures 1b and e, respectively). Three solvent molecules are included in the cavity at the center of the tripod structure. $\mathrm{IE}_{20^{-}} 1$ exhibited both wavy and tripod structures in a biphasic separation, as shown in Figures 1c and $\mathrm{d}$. The steric repulsion of terminal methyl groups has been reported to be one of the plausible factors for the emergence of the odd-even effect. $^{37}$ If IE-1 with odd numbers of carbon atoms in the alkyl chain unit assumes the tripod structure, steric repulsion of the terminal methyl groups occurs among the building blocks. In contrast, the adsorption energy of the tripod structure appears to be more advantageous than that of the wavy structure because of the differences in the number of alkyl chains per building block attached to the HOPG surface. Therefore, the phase-separated wavy and tripod structures in $\mathrm{IE}_{20^{-}}-1$ can be formed due to the equilibrium between the tripod structures with steric repulsions at the alkyl chain end and the wavy structures with lower adsorption energies of alkyl chains. Thus, the $2 \mathrm{D}$ structures of $\mathrm{IE}_{n}-1$ ( $n=18-21)$ change alternately on the basis of the odd-even alkyl chain-length effect.

After the TCR, all of the 2D structures of IEn-2 were converged into the same linear structures, irrespective of the alkyl chain length (Figure 1f). In the linear structure, the alkyl chains were interdigitated. Indeed, the positions of the intramolecular hydrogen bonds were altered after the TCR. In $\mathrm{IE}_{n}-1$, before TCR, hydrogen bonds exist between the isobutenyl ether oxygen atom and the amide $\mathrm{NH}$ proton and between the $4-\mathrm{H}$ naphthoate proton and the carbonyl oxygen atom. After the TCR, the conversion from ether to hydroxyl groups resulted in the formation of new intramolecular hydrogen bonding between hydroxyl and carbonyl groups in $\mathrm{IE}_{n}-2$. However, this is not viewed as the main reason for the drastic $2 \mathrm{D}$ structural transformation. In addition to changes in the intramolecular hydrogen bonds, TCR caused the shortening of the intramolecular distance of naphthalene units, and the naphthalene units of $\mathrm{IE}_{n}-2$ have to flip in opposite directions due to the steric repulsions. Consequently, the molecule of $\mathrm{IE}_{n}-2$ forms an extended linear structure. Thus, the oddeven effect found in $\mathrm{IE}_{n}-1(n=18-21)$ was canceled in $\mathrm{IE}_{n}-2$, possibly because of the limitation of the intramolecular distance of the naphthalene units.
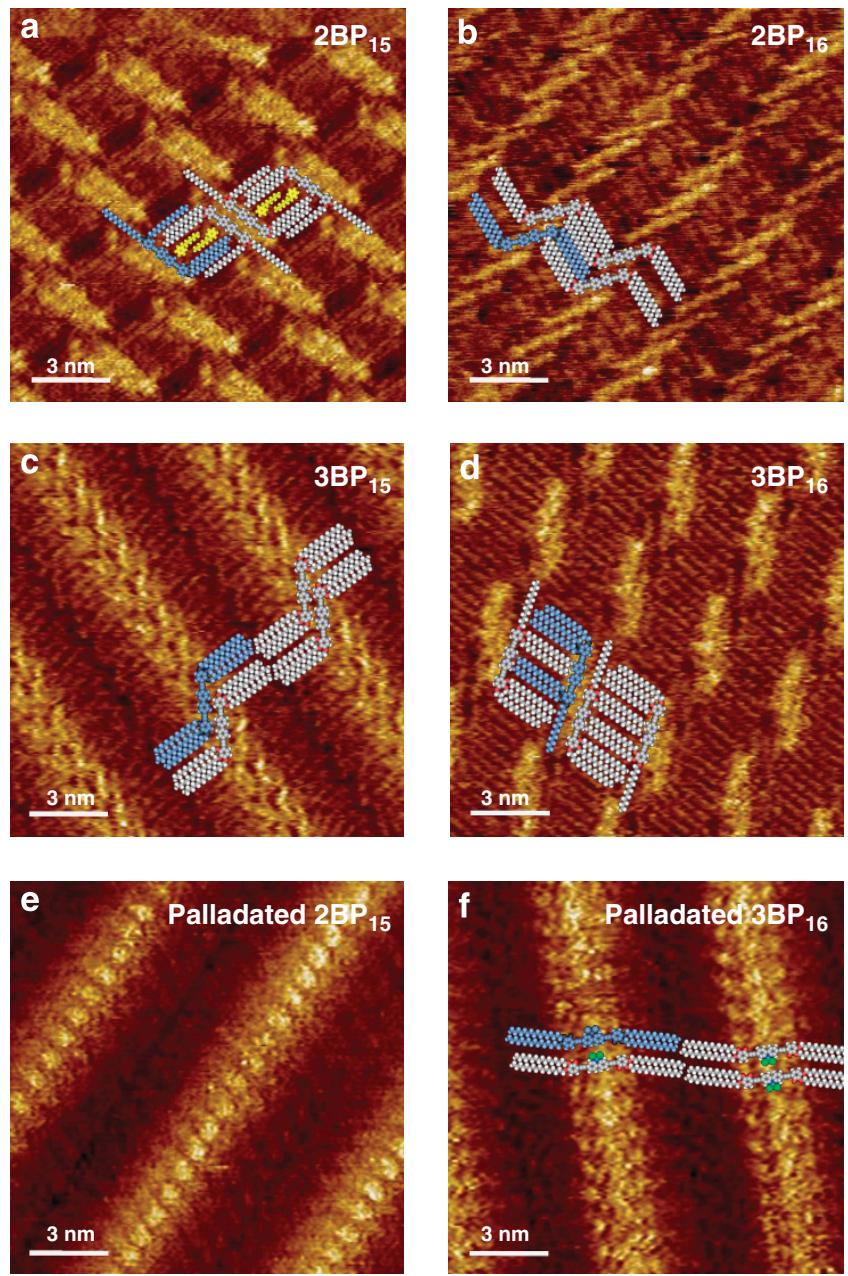

Figure 2 Selected STM images of double and triple alkyl chain-substituted $\mathrm{BP}_{n}(n=15$ and 16) and palladated samples at the HOPG/1-phenyloctane interface. Molecular models are superimposed on each STM image. Labels and tunneling conditions: (a) $2 \mathrm{BP}_{15}, I=1.8 \mathrm{pA}, V=-292 \mathrm{mV}$; (b) $2 \mathrm{BP}_{16}$, $I=2.5 \mathrm{pA}, V=-272 \mathrm{mV}$; (c) $3 \mathrm{BP}_{15}, I=1.2 \mathrm{pA}, V=-275 \mathrm{mV}$; (d) $3 \mathrm{BP}_{16}$, $I=1.1 \mathrm{pA}, \quad V=-660 \mathrm{mV}$; (e) palladated $2 \mathrm{BP}_{15}, I=1.5 \mathrm{pA}, V=-620 \mathrm{mV}$; and (f) palladated $3 \mathrm{BP}_{16}, I=1.3 \mathrm{pA}, V=-654 \mathrm{mV}$.

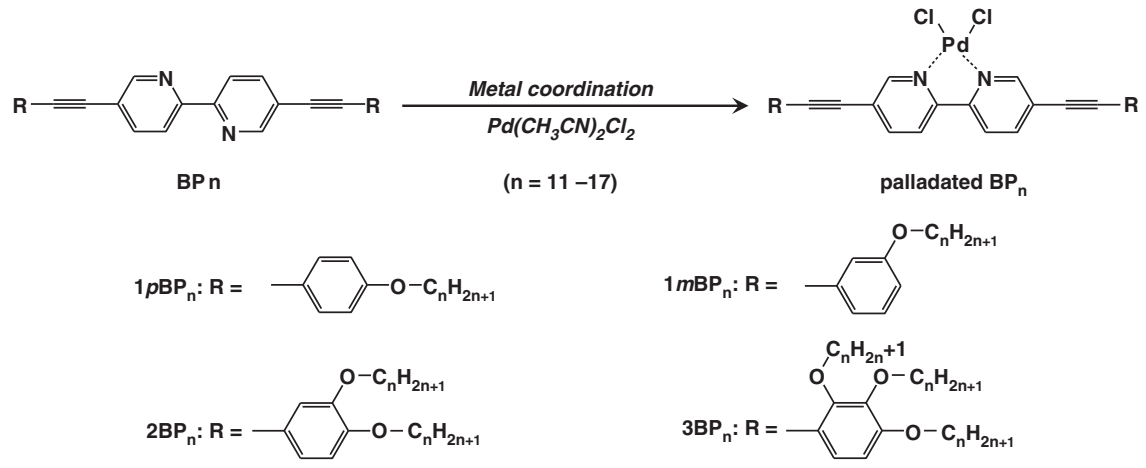

Scheme 2 Chemical structures of bipyridine derivatives substituted by different numbers and lengths of alkyl chains $\left(\mathrm{BP}_{n}\right)$ and those of palladated $\mathrm{BP}_{n}$. 


\section{ODD-EVEN EFFECT AND METAL COORDINATION}

In this section, the number and length of alkyl chain units were studied. As bipyridine is able to coordinate a metal, bipyridine derivatives with different numbers and lengths of alkyl tails $\left(\mathrm{BP}_{n}\right.$, $n=11-17$; Scheme 2) were synthesized to study the odd-even alkyl chain-length effect and the effect of metallation on the formation of 2D structures at the HOPG/1-phenyloctane interface. ${ }^{33-35}$

The $\mathrm{BP}_{n}$ substituted with a single alkyl chain at the para and meta positions on each side $\left(1 \mathrm{pBP}_{n}\right.$ and $\left.1 \mathrm{mBP}_{n}\right)$ showed no odd-even effect on the $2 \mathrm{D}$ structures. In contrast, the nanostructures of double $\left(2 \mathrm{BP}_{n}\right)$ and some triple alkyl chain-substituted $\mathrm{BP}_{n}\left(3 \mathrm{BP}_{n}\right)$ molecules were alternately altered due to odd-even effects. Figures $2 \mathrm{a}$ and $\mathrm{b}$ show the STM images of $2 \mathrm{BP}_{n}$. The $\pi$-conjugated bipyridine core was observed as a region of bright contrast, whereas the alkyl chain units were included in the dark region in the STM image. The $2 \mathrm{BP}_{\text {even }}$ adopted a Z-shaped morphology, and the alkyl chains were interdigitated. In the case of odd carbon numbers in the alkyl chain unit $\left(2 \mathrm{BP}_{\text {odd }}\right)$, a pair of L-shaped molecules aligned in a columnar manner upon accommodation of the alkyl chains between the columns.

The $3 \mathrm{BP}_{n}$ with $n=11-13$ showed no odd-even alkyl chain-length effect, whereas that with $n=14-17$ formed two types of molecular arrangements depending on the alkyl chain length (Figures $2 \mathrm{c}$ and d). Therefore, a specific alkyl chain-length range exists in the $3 \mathrm{BP}_{n}$ in which the odd-even effect is exhibited. The odd-even effect has been explained in terms of the steric repulsion of terminal methyl groups. ${ }^{37}$ The solid/liquid interface system features molecule-molecule and molecule-substrate interactions that form the $2 \mathrm{D}$ structures. Therefore, we suggest that the odd-even effects emerge when the $2 \mathrm{D}$ structure formation is dominated by the intermolecular interactions of the alkyl chain units.

One of the advantages of a solid/liquid interface system is that post-metallation can be applied in situ through the addition of $\mathrm{Pd}\left(\mathrm{CH}_{3} \mathrm{CN}\right)_{2} \mathrm{Cl}_{2}$ solution to an already-existing sample drop on HOPG for STM imaging. The metallation in the solution phase might be more favorable than that on the substrate; therefore, the gradual replacement of $\mathrm{BP}_{n}$ and metallated molecules could be observed. Although in situ metallation on a HOPG surface is possible, as previously mentioned, ex situ metallation for a series of $\mathrm{BP}_{n}$ samples was performed separately to compare the $2 \mathrm{D}$ structural features between $\mathrm{BP}_{n}$ and the metallated samples. After $2 \mathrm{BP}_{n}$ and $3 \mathrm{BP}_{n}$ were palladated, they formed the same lamellar structures without interdigitation of their alkyl chains; specifically, the oddeven effect was canceled (Figures $2 \mathrm{e}$ and $\mathrm{f}$ ). In the case of $3 \mathrm{BP}_{n}$, one of the alkyl chains, possibly at the o-position, is dangling into the solvent phase. The metal coordination changed the molecular width at the bipyridine core, and the molecular width of the bipyridine unit with $\mathrm{PdCl}_{2}$ became almost the same as that of the peripheral alkyl chain unit. In addition, the intermolecular interaction of the bipyridine unit with $\mathrm{PdCl}_{2}$ was introduced into the system. Thus, differences in the intermolecular interactions accounted for the $2 \mathrm{D}$ structure formation of $\mathrm{BP}_{n}$ monolayers.

\section{HOST-GUEST CHEMISTRY AND PHOTODIMERIZATION}

Anthracene can photodimerize under UV irradiation, which results in the formation of a photodimer connected by covalent bonds due to a $(4+4)$ cycloaddition. ${ }^{40,41}$ Consequently, a third dimension would be added to the $2 \mathrm{D}$ monolayer system. In this context, we synthesized an anthracene derivative (AN-1) that contained decyloxy chains (Scheme 3) and performed both in situ and ex situ photodimerization experiments. ${ }^{36}$
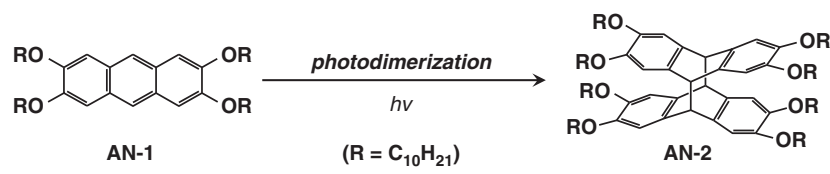

Scheme 3 Chemical structures of the anthracene derivative (AN-1) and its photodimer (AN-2)
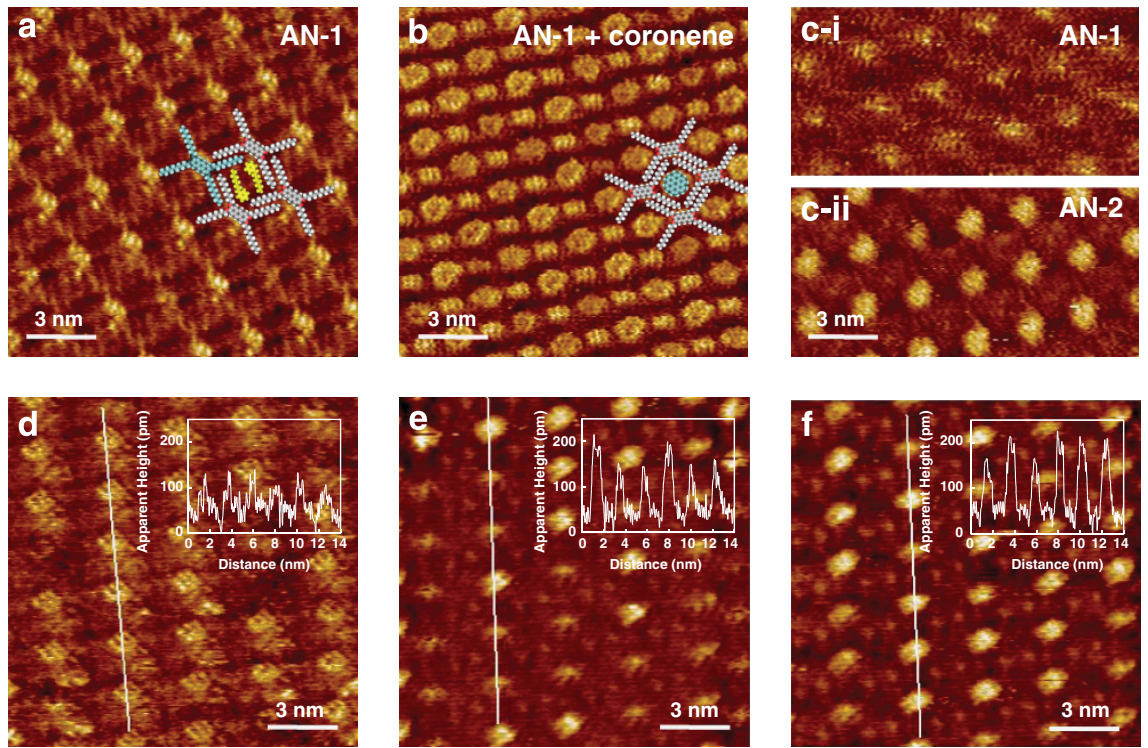

Figure 3 STM images of AN-1 (a, ci), AN-1 with coronene (b) and photodimer AN-2 (cii). Molecular models are depicted on the basis of the STM images. (d, e) The consecutive STM images during in situ photodimerization at the HOPG/1-phenyloctane interface; the images were recorded in a similar area. (d) AN-1 before UV irradiation at $365 \mathrm{~nm}$. e was obtained just after the UV irradiation for $30 \mathrm{~min}$, whereas further scanning without UV light provided f. Tunneling conditions: (a) $I=1.2 \mathrm{pA}, V=-265 \mathrm{mV}$; (b) $I=1.0 \mathrm{pA}, V=-414 \mathrm{mV}$, (ci and cii): $I=1.0 \mathrm{pA}, V=-1000 \mathrm{mV}$. STM observations in d, e were performed under the same tunneling conditions: $I=5.0 \mathrm{pA}, V=-1000 \mathrm{mV}$. 
Figure 3a shows the STM images of AN-1 at the HOPG/1phenyloctane interface. Three bright dots were derived from the anthracene moiety because molecular orbital calculations using the extended Hückel method revealed that three charge density contours of the highest occupied molecular orbital (HOMO) are localized in association with aromatic rings. The alkyl chain units of AN-1 formed a porous network structure filled with solvent molecules. These cavities were used as the template for host-guest chemistry to demonstrate the fabrication of a multi-functional system through the assembly of other components. An excess amount of coronene was added to the initial drop on the HOPG. STM observation revealed that bright disks are incorporated into the cavities, which suggests the co-adsorption of the coronene between rows of the threespotted AN-1 (Figure 3b).

Photodimerization of the anthracene derivative was performed in situ and ex situ. First, ex situ photodimerization was performed in a flask under UV irradiation at $365 \mathrm{~nm}$, and the reaction was checked using the UV-visible spectroscopy and nuclear magnetic resonance. Then, STM observations were performed at the HOPG/1-phenyloctane interface. Although the lattice constants of the photodimer AN-2 were almost identical to those of AN-1, the image contrast of AN-2 (Figure 3cii) was quite different from that of AN-1 (Figure 3ci) under the same tunneling conditions. The cross-sectional analysis indicates that the apparent height of AN-2 is greater than that of AN-1, which suggests the occurrence of changes in not only the physical height but also the electron density of the states. Thus, the monomer AN-1 and the photodimer AN-2 on HOPG can apparently be discriminated via the STM image contrast.

Second, in situ photodimerization was conducted at solid/liquid interface in a STM setup (Figures 3d-f). After the observation of AN1 (Figure 3d), UV irradiation was directly applied to the 1-phenyloctane solution of AN-1 deposited on HOPG. Only 30 min of UV irradiation revealed a mixture of monomer $\mathrm{AN}-1$ and photodimer AN-2 (Figure 3e). The AN-2 subsequently became the major component in the STM image after an additional $10 \mathrm{~min}$ of STM scanning (Figure 3f). In the present solid/liquid interface system, the reaction can preferentially occur in the solution phase rather than on the substrate. As the photodimerization proceeds, the original adsorbates of $\mathrm{AN}-1$ are replaced by the increasing numbers of AN-2 products due to the equilibrium. Thus, the photodimerization and the subsequent adsorption/desorption of $\mathrm{AN}-1$ and $\mathrm{AN}-2$ were visualized in situ.

\section{CONCLUSIONS AND OUTLOOK}

We have presented a summary of our recent work on the formation of 2D structure, especially those formed by van der Waals interactions and including the odd-even effect of alkyl chains. Although preprogramming of the tectons with a directional interaction site is a common and powerful method to produce regular $2 \mathrm{D}$ structures, the odd-even effect can also be helpful in the construction of the complicated nanoarchitectures, which is difficult to achieve using lithographic techniques. Submolecularly resolved STM images at the solid/liquid interface revealed the 2D structural transformations induced by external stimuli, such as heat, metallation and UV irradiation.

Our efforts to explore the multicomponent system and bottom-up fabrication of surface-based 3D structures toward the use of nanofunctions in a real space will continue. We believe that post-tunable nanopatterned surfaces based on the concepts of supramolecular chemistry inspired by the living system have great potential to result in the construction of an intelligent molecular system.

\section{ACKNOWLEDGEMENTS}

This work has been partially supported by a grant from the Sumitomo Foundation, JSPS (23750137) and MEXT KAKENHI (21106522; 23106722). I am very grateful to all of the collaborators: Dr M Kanesato, Professor K Hiratani, Dr E Koyama, Dr S Tsuzuki, Dr H Kihara, Dr H Tokuhisa, Dr K Miyake, Dr K Omori, Ms K Fujiwara, Ms M Takahashi (AIST, Japan), Professor J-M Lehn (Strasbourg, France) and Professor T S Balaban (Marceille, France).

1 Miyazawa, A., Fujiyoshi, Y. \& Unwin, N. Structure and gating mechanism of the acetylcholine receptor pore. Nature 423, 949-955 (2003).

2 Unwin, N. Refined Structure of the nicotinic acetylcholine receptor. J. Mol. Biol. 346, 967-989 (2005).

3 Lehn, J-M. Toward self-organization and complex matter. Science 295, 2400-2403 (2002).

4 Whitesides, G. M. \& Grzybowski, B. Self-assembly at all scales. Science 295, 2418-2421 (2002).

5 Barth, J. V., Costantini, G. \& Kern, K. Engineering atomic and molecular nanostructures at surfaces. Nature 437, 671-679 (2005).

6 Gomar-Nadal, E., Puigmartí-Luis, J. \& Amabilino, D. B. Assembly of Functional molecular nanostructures on surfaces. Chem. Soc. Rev. 37, 490-504 (2008).

7 Hosseini, M. W. Self-Assembly and generation of complexity. Chem. Commun. 5825-5829 (2005).

8 De Feyter, S. \& De Schryver, F. C. Two-dimensional supramolecular selfassembly probed by scanning tunneling microscopy. Chem. Soc. Rev. 32, 139-150 (2003).

9 Plass, K. E., Grzesiak, A. L. \& Matzger, A. J. Molecular packing and symmetry of twodimensional crystals. Acc. Chem. Res. 40, 287-293 (2007).

10 De Feyter, S. \& De Schryver, F. C. Self-assembly at the liquid/solid interface: STM reveals. J. Phys. Chem. B. 109, 4290-4302 (2005).

11 Elemans, J. A. A. W., Lei, S. \& De Feyter, S. Molecular and Supramolecular networks on surfaces: from two-dimensional crystal engineering to reactivity. Angew. Chem. Int. Ed. 48, 7298-7332 (2009).

12 Palma, C. -A., Ciesielski, A., Bonini, M. \& Samorì, P. in Functional supramolecular architectures (eds Samorì, P. \& Cacialli, F.) Ch. 13, 423-455 (Wiley-VCH Verlag \& Co. KGaA, Weinheim, Germany, 2011).

13 Otsuki, J. STM studies on porphyrins. Coord. Chem. Rev. 254, 2311-2341 (2010).

14 Lackinger, M., Griessl, S., Kampschulte, L., Jamitzky, F. \& Heckl, W. M. Dynamics of grain boundaries in two-dimensional hydrogen-bonded molecular networks. Small 1 , 532-539 (2005).

15 Surin, M., Samorì, P., Jouaiti, A., Kyritsakas, N. \& Hosseini, M. W. Molecular tectonics on surfaces: bottom-up fabrication of 1D coordination networks that form $1 D$ and 2D arrays on graphite. Angew. Chem. Int. Ed. 46, 245-249 (2006).

16 Ciesielski, A., Piot, L., Samorì, P., Jouaiti, A. \& Hosseini, M. W. Molecular tectonics at the solid/liquid interface: controlling the nanoscale geometry, directionality, and packing of 1D coordination networks on graphite surfaces. Adv. Mater. 21, 1131-1136 (2009).

17 Madueno, R., Räisänen, M. T., Silien, C. \& Buck, M. Functionalizing hydrogenbonded surface networks with self-assembled monolayers. Nature 454, 618-621 (2008).

18 Chen, T., Wang, D., Zhang, X., Zhou, Q. -L., Zhang, R. -B. \& Wan, L. -J. In situ scanning tunneling microscopy of solvent-dependent chiral patterns of 1,4-Di[4- $\mathrm{N}$-(trihydroxymethyl)methyl carbamoylphenyl]-2,5-didodecyloxybenzene molecular assembly at a liquid/highly oriented pyrolytic graphite interface. J. Phys. Chem. C 114, 533-538 (2010).

19 Tahara, K., Furukawa, S., Uji-i, H., Uchino, T., Ichikawa, T., Zhang, J., Mandouh, W. Sonoda, M., De Schryver, F. C., De. Feyter, S. \& Tobe, Y. Two-dimensional porous molecular networks of dehydrobenzo[12]annulene derivatives via alkyl chain interdigitation. J. Am. Chem. Soc. 128, 16613-16625 (2006).

20 Yang, Y. \& Wang, C. Solvent effects on two-dimensional molecular self-assemblies investigated by using scanning tunneling microscopy. Curr. Opin. Colloid Interface Sci. 14, 135-147 (2009).

21 Hwang, R. Q. \& Bartelt, M. C. Scanning tunneling microscopy studies of metal on metal epitaxy. Chem. Rev. 97, 1063-1082 (1997).

22 Coratger, R., Sivel, V., Ajustron, F. \& Beauvillain, J. Scanning tunneling microscopy and related techniques for surface analysis. Micron 25, 371-385 (1994).

23 Kubby, J. A. \& Boland, J. J. Scanning tunneling microscopy of semiconductor surfaces. Surf. Sci. Rep. 26, 61-204 (1996).

24 Cheng, S. Scanning tunneling microscopy imaging of small adsorbed molecules on metal surfaces in an ultrahigh vacuum environment. Chem. Rev. 97, 1083-1096 (1997).

25 Smith, R. K., Lewis, P. A. \& Weiss, P. S. Patterning self-assembled monolayers. Prog. Surf. Sci. 75, 1-68 (2004).

26 Poirier, G. E. Characterization of organosulfur molecular monolayers on Au(111) using scanning tunneling microscopy. Chem. Rev. 97, 1117-1128 (1997).

27 Cyr, D. M., Venkataraman, B. \& Flynn, G. W. STM investigations of organic molecules physisorbed at the liquid-solid interface. Chem. Mater. 8, 1600-1615 (1996). 
28 Samorì, P. \& Rabe, J. P. Scanning probe microscopy explorations on conjugated (macro)molecular architectures for molecular electronics. J. Phys. Condens. Matter 14 9955-9973 (2002).

29 Piot, L., Bonifazi, D. \& Samorì, P. Organic reactivity in confined spaces under scanning tunneling microscopy control: tailoring the nanoscale world. Adv. Funct. Mater. 17 3689-3693 (2007).

30 Uemura, S., Tanoue, R., Yilmaz, N., Ohira, A. \& Kunitake, M. Molecular dynamics in twodimensional supramolecular systems observed by STM. Materials 3, 4252-4276 (2010).

31 Omori, K., Kikkawa, Y., Tokuhisa, H., Kanesato, M. \& Hiratani, K. Transformation of two-dimensional self-assembled structures of noncycric isobuteny amide compounds synthesized via tandem claisen rearrangement. Colloids Surf. A: Physicochem. Eng. Asp. 356, 58-62 (2010).

32 Omori, K., Kikkawa, Y., Kanesato, M. \& Hiratani, K. Fabrication and transformation of novel two-dimensional tripod structures: structural modulation by alkyl chain length and tandem claisen rearrangement. Chem. Commun. 46, 8008-8010 (2010).

33 Kikkawa, Y., Koyama, E., Tsuzuki, S., Fujiwara, K., Miyake, K., Tokuhisa, H. \& Kanesato, M. Two-dimensional nanostructure control by molecular width variation with metal coordination. Langmuir 22, 6910-6914 (2006).

34 Kikkawa, Y., Koyama, E., Tsuzuki, S., Fujiwara, K., Miyake, K., Tokuhisa, H. \& Kanesato, $M$. Odd-even effect and metal induced structural convergence in self-assembled monolayers of bipyridine derivatives. Chem. Commun. 1343-1345 (2007).
35 Kikkawa, Y., Koyama, E., Tsuzuki, S., Fujiwara, K. \& Kanesato, M. Bipyridine derivatives at solid/liquid interface: effect of the number and length of peripheral alkyl chains. Langmuir 26, 3376-3381 (2010).

36 Kikkawa, Y., Kihara, H., Takahashi, M., Kanesato, M., Balaban, T. S. \& Lehn, J. -M. Two-dimensional structures of anthracene derivatives: photodimerization and hostguest chemistry. J. Phys. Chem. B. 114, 16718-16722 (2010).

37 Tao, F. \& Bernasek, S. L. Understanding odd-even effects in organic self-assembled monolayers. Chem. Rev. 107, 1408-1453 (2007).

38 Albrecht, M., Osetska, R. \& Froehlich, R. The hiratani-double-claisen rearrangement as a key step in the preparation of sequential Bis(8-hydroxyquinoline) ligands. Synlett 6, 924-926 (2006).

39 Hiratani, K. \& Albrecht, M. The tandem claisen rearrangement in the construction of building blocks for supramolecular chemistry. Chem. Soc. Rev. 37, 2413-2421 (2008).

40 Bouas-Laurent, H., Castellan, A., Desvergne, J. -P. \& Lapouyade, R. Photodimerization of anthracenes in fluid solution: structural aspects. Chem. Soc. Rev. 29, 43-55 (2000).

41 Bouas-Laurent, H., Castellan, A., Desvergne, J. -P. \& Lapouyade, R. Photodimerization of anthracenes in fluid solutions: (Part 2) mechanistic aspects of the photocycloaddition and of the photochemical and thermal cleavage. Chem. Soc. Rev. 30, 248-263 (2001).

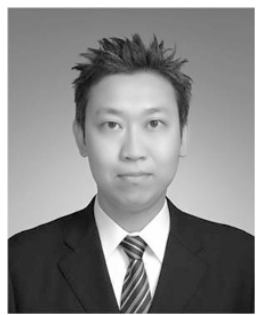

Yoshihiro Kikkawa is currently a researcher in National Institute of Advanced Industrial Science and Technology (AIST), Japan. He was born in Kochi, Japan, in 1975. He received his Bachelor Degree (1998), Master Degree (2000) from Science University of Tokyo, and Doctorate Degree (2002) from Tokyo Institute of Technology, Japan. He was a postdoctoral researcher of Solution Oriented Research for Science and Technology (SORST), Japan Science and Technology Agency (2002) and a special postdoctoral researcher in Polymer Chemistry Laboratory, RIKEN Institute (2003). From 2005, he has been working at AIST. His research interests cover selfassembly at surfaces, scanning probe microscopy, and biodegradable polymers. He published 60 original research papers, 3 reviews and 2 book chapters. He received the Award for Encouragement of Research in Polymer Science from the Society of Polymer Science, Japan (2010). 